\title{
Innovación en gestion de costes: del abc al tdabc
}

\section{Innovation in cost management: from abc to tdabc}

\author{
Patxi Ruiz de Arbulo Lopez ' y Jordi Fortuny-Santos 2 \\ I Dpto. de Organización de Empresas. Escuela Técnica Superior de Ingeniería de Bilbao. \\ Universidad del País Vasco. Alda Urquijo s/n. 48013 Bilbao. \\ 2 Dpto. de Organización de Empresas. Escuela Politécnica Superior de Ingeniería de Manresa. \\ Universidad Politécnica de Cataluña. Av. Bases de Manresa, 61 -73, 08242, Manresa, Barcelona. \\ patxi.ruizdearbulo@ehu.es Jordi.Fortuny@upc.edu
}

\begin{abstract}
Fecha de recepción: 14-05-2010
Fecha de aceptación: 28-07-2010
\end{abstract}

Resumen: Este artículo analiza la aplicabilidad del método Time-driven activity-based costing (TDABC) en la empresa industrial. En las últimas décadas, las empresas que han adoptado innovaciones en la gestión de la producción (por ejemplo, lean manufacturing) han sentido la necesidad de buscar nuevos métodos de control de costes. Con este fin nació, entre otros, el sistema de costes basados en las actividades ( $A B C$ ), cuya aplicación no está exenta de inconvenientes. Recientemente, se ha desarrollado el método TDABC que ya se emplea en alguna empresa española. Las conclusiones del trabajo muestran como el sistema TDABC recoge la heterogeneidad de los procesos mejor que el sistema $A B C$.

Palabras clave: Coste basado en las actividades (ABC), Time-driven activity-based costing (TDABC), Contabilidad de costes, Producción ajustada.

\begin{abstract}
This paper analyses the applicability of Time-driven activity-based costing (TDABC) in manufacturing companies. In the last decades, companies introducing new manufacturing techniques (like lean manufacturing) have felt the need for new accounting methods. Activity-based costing ( $A B C)$, amongst others, was created to support these requirements. But $A B C$ has also met with difficulties. Thus, a new approach termed TDABC, which is already implemented in some Spanish factories, has been developed. This paper shows how TDABC can capture the heterogeneity of manufacturing activities better that $A B C$.
\end{abstract}

Key words: Activity-based costing (ABC), Time-driven activity-based costing (TDABC), Cost accounting, Lean manufacturing.

\section{Introducción}

Los últimos 25 años se han caracterizado por cambios en el entorno empresarial como el aumento de la competencia o las cada vez mayores exigencias de los clientes en términos de calidad, precio, personalización y plazos de entrega. Para darles respuesta, se han introducido innovaciones en los sistemas productivos, como por ejemplo, lean manufacturing (Marín y Delgado, 2000).

De modo análogo, se ha observado que la contabilidad de costes tradicional evalúa desfavorablemente innovaciones introducidas por los nuevos enfoques en gestión de la producción (Iglesias, 2004). Por ejemplo, en La Meta (Goldratt y Cox, 2005), los autores de la teoría de las limitaciones exponen que «la contabilidad es el principal enemigo de la producción». Esto se debe a que la contabilidad de costes nació para dar soporte a un entorno basado en la producción en masa con sistemas productivos donde el coste de mano de obra directo era muy importante (Tatikonda, 1988). En la actualidad, ésta no es la situación de muchas empresas. Esta disfunción de los métodos de contabilidad de costes tradicionales ha propiciado la aparición de nuevos sistemas que se adapten al nuevo entorno competitivo y a los nuevos sistemas de organizar la producción.

En la década de los 80, ante la desconfianza que suscitaba la información derivada de los sistemas tradicionales (Kaplan 1983 y 1984), Robin Cooper y Robert Kaplan desarrollaron la metodología de costes ABC (coste basado en las actividades). El sistema 
ABC fue diseñado para resolver la asignación de los costes indirectos - cada vez más importantes en las empresas- que se realizaba de modo casi arbitrario.

El sistema $A B C$ sostiene que los productos no consumen directamente recursos, sino actividades y son éstas las que, a su vez, consumen los recursos y causan los costes. Igual que en el método full costing, es preciso localizar los costes de los departamentos o centros de costes. El sistema ABC, en lugar de asignar los costes indirectos a los productos, dichos costes se asignan a actividades realizadas. Se denomina actividades a las tareas principales o auxiliares cuya realización supone un coste. Después, se asignan los costes de las actividades a los productos y otros objetos de coste (Kaplan y Cooper, 1988), tal como se muestra en la Figura I, por medio de portadores o inductores relacionados con la generación de dichos costes.

Figura 1

Modelo de Costes basados en las actividades

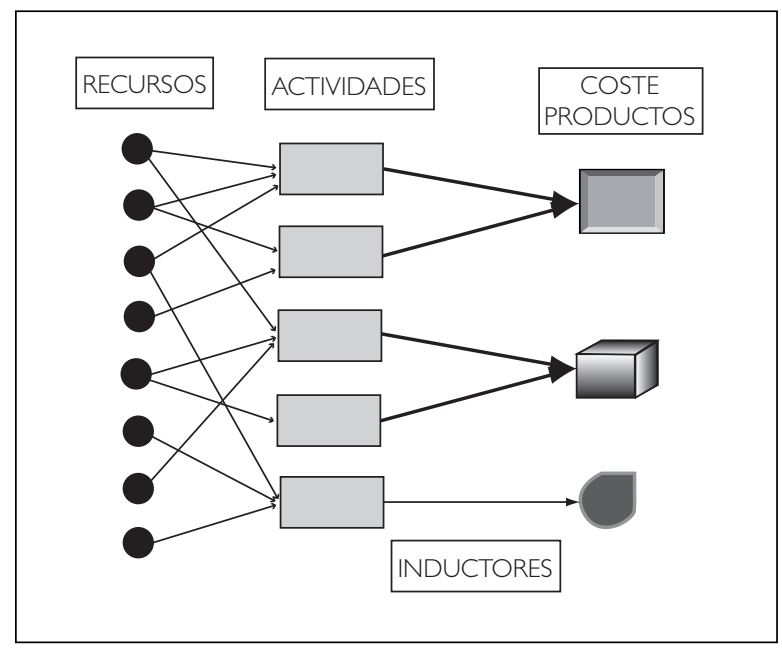

Fuente: Elaboración propia

En el método ABC, la imputación de los costes es más realista (Mallo y Jiménez, 2000) y permite a la empresa saber cuánto le cuestan las actividades que desarrolla. A partir de esta información, la empresa puede eliminar actividades sin valor añadido y mejorar la ejecución de las actividades para reducir sus costes. Esta actitud coincide con los principios de la producción ajustada o lean manufacturing (Ripoll y Tamarit, 1996). Como inconveniente, el sistema ABC tampoco está exento de subjetividad, pues el personal debe hacer estimaciones sobre su dedicación horaria a una u otra tarea y también hay que adoptar criterios de reparto de los costes indirectos en- tre las actividades. Además, ABC introduce grandes costes administrativos e informáticos (Mallo y Jiménez, 2000).

En el año 2004, Robert Kaplan y Steven Anderson desarrollan un nuevo enfoque de ABC (Kaplan y Anderson, 2004 y 2007) Ilamado Time-driven ABC (TDABC) con el fin de superar algunas dificultades del sistema de costes $A B C$.

\section{Inconvenientes de los sistemas de costes basados en volumen}

Los métodos tradicionales de costes (también Ilamados sistemas basados en volumen) clasifican los costes en directos o indirectos. Los primeros los asignan directamente a los productos en función del consumo que cada producto realiza.

Con respecto a los costes indirectos, su imputación se realiza en dos etapas:

- En la primera etapa, se distribuyen los recursos consumidos entre las secciones o centros de coste. Asimismo, en esta primera etapa, los costes acumulados en las secciones auxiliares se distribuyen entre las secciones principales en función del uso que estas últimas hayan hecho de las primeras.

- En la segunda etapa, se asignan los costes acumulados en las secciones principales a los productos. Esta operación se lleva a cabo mediante las denominadas unidades de obra, que relacionan las secciones o centros de coste con el uso que los productos hacen de ellas.

Con este sistema se comete un doble error:

- Enmascarar en un centro de coste el consumo de factores de actividades no relacionadas con el centro.

- Evaluar todo el consumo de factores de un centro de coste por la medición de una sola de las actividades desarrolladas.

Habitualmente las unidades de obra elegidas (horashombre, horas-máquina) guardan una alta relación con el volumen de producción de cada producto. Con esta forma de reparto los costes indirectos se asignan en base al volumen de producción de cada producto y no necesariamente tiene que ser así, ya que productos de baja producción pueden requerir muchas actividades que son las que originan dichos costes. 
Debido a su origen, este sistema es válido para empresas con sistemas productivos de un solo producto o con gamas muy reducidas de unidades estandarizadas y donde la mano de obra sea un componente fundamental de la estructura de costes (Tirado, 2003).

\section{Revisión del sistema de costes $A B C$}

Se desarrolló el método $\mathrm{ABC}$ como respuesta a los problemas de asignación de los costes indirectos de los sistemas de costes basados en volumen.

El sistema de costes $A B C$ primero identifica las actividades que tiene lugar en la empresa (Mallo y Jiménez, 2000). A continuación, localiza las actividades en las secciones o centros de coste de la empresa. Una actividad puede desarrollarse en más de un centro. Los costes directos se asignan directamente a los productos. El siguiente paso es localizar las cargas indirectas en cada sección y asignarlas a las actividades.

En algunos casos, se identifica fácilmente qué actividad ha generado dicho coste y su imputación es directa, pero en otros casos se genera una bolsa de coste indirecto en la sección que tiene que repartirse entre las actividades por medio de algún criterio. Para cada actividad hay que asignar un portador de costes o inductor (en inglés, cost driver). Un inductor es la unidad de una actividad que causa cambios en el coste de dicha actividad. El inductor realiza la función de unidad de medida de la actividad, por lo que debe estar directamente relacionado con los recursos consumidos. Tanto puede ser un input de la actividad, un output u otro indicador físico de la misma. El coste total de cada actividad se divide para hallar el coste unitario del inductor (motivo por el que esta técnica se denomina también rate-based $\mathrm{ABC}$ ). A partir de ahí, el coste de cada producto u objeto de coste se obtiene en función del consumo de unidades de inductor (Kaplan y Cooper, 1988), más los costes directos correspondientes, tal como se muestra en la Figura 2.

El sistema $A B C$ no ha sido aceptado por todos (Rigby, 2003). Mediante el ejemplo de la tabla I, que representa las actividades de un almacén relacionadas con la recepción de materiales, expondremos las razones principales de la baja aceptación del método $A B C$ (Kaplan y Anderson, 2004).

I. La puesta en marcha de un sistema $A B C$ es un proceso muy largo, puesto que el desarrollo de entrevistas y encuestas necesarias para llegar a comprender las actividades que se realizan en la empresa exige mucho tiempo. Por ejemplo, supongamos que el departamento de recepción de materiales realiza las 6 actividades que aparecen en la primera columna de la Tabla I. Cuando al responsable del mismo se le pregunta cuantos recursos y tiempo dedica a cada una de las actividades responde con los porcentajes de la segunda columna.

2. Las empresas cuestionan la forma en que se realiza la asignación de los costes a las actividades porque muchas veces se basan en cálculos subjetivos que cada responsable hace sobre el por-

Figura 2

Metodología de imputación del coste según el sistema $A B C$

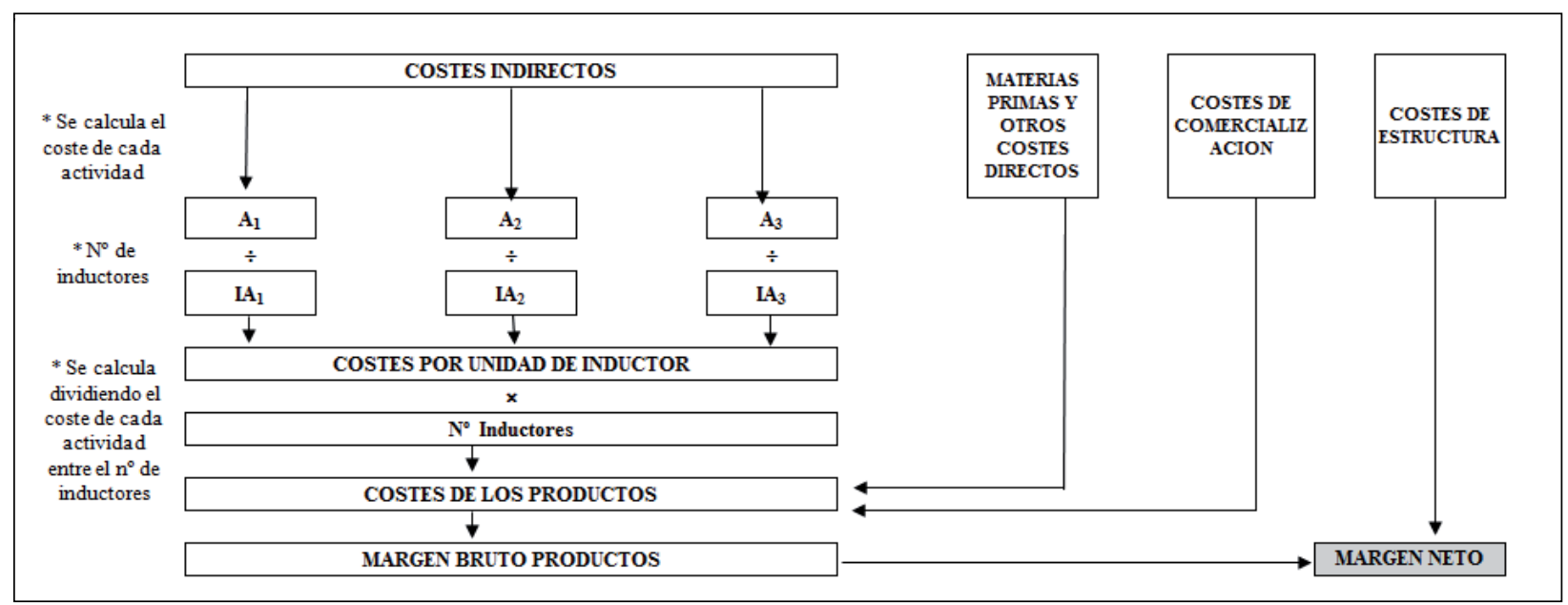

Fuente: Basado en Baguer y De Zárraga (2002) 
centaje de su tiempo que dedica a cada actividad. En el ejemplo de la Tabla I, son los valores de la segunda columna.

3. Cuando se realizan las encuestas, muy pocos responsables informan del porcentaje de tiempo improductivo o no utilizado $y$, por lo tanto, se calculan los inductores de coste como si se trabajase a capacidad total.

4. El modelo $A B C$ no es lo bastante preciso como para permitir capturar la complejidad de las operaciones reales de una empresa. Para tener en cuenta todos los tipos de recepciones de materiales que se pueden dar, es necesario añadir al sistema $A B C$ muchas actividades, lo que hace, por una parte, que aumente su complejidad y, por otra, que se incremente su subjetividad en la estimación del tiempo que los responsables dedican a cada una de las actividades. En la quinta columna del ejemplo de la Tabla I, se considera que el coste de la actividad de «puntear albarán de entrega con pallet» siempre es el mismo, independientemente de que sea una paleta con mercancía de un solo producto o de múltiples productos, cuando seguramente una tome más tiempo que otra y con ello consuma más cantidad de recursos (en este caso, tiempo del operario).

En la operativa de la tabla I, el coste mensual de la actividad «puntear albarán de entrega con pallet» asciende a la cantidad de $7.500 €$. Si se toma como in- ductor de esta actividad el número de paletas y su valor es 2.000 paletas al mes, en el sistema de costes $A B C$ el coste de «puntear albarán de entrega con paletas es de $3,75 €$ por paleta (cociente de $7.500 €$ entre 2.000 paletas). Para otras actividades del ejemplo, los inductores pueden ser el número de entregas o el número de camiones (200 camiones en el ejemplo de la Tabla I).

Para superar los inconvenientes del sistema de costes ABC, el mismo Robert Kaplan junto a Steven Anderson desarrollaron el sistema llamado Time-driven ABC (TDABC) (Kaplan y Anderson, 2004 y 2007), que se expone a continuación.

\section{Time-driven ABC}

La metodología TDABC asigna los recursos a las actividades tal como se muestra en el apartado B de la Figura 3. El enfoque TDABC identifica los diferentes grupos de recursos o departamentos, sus costes y su capacidad normal. Por ejemplo, para el departamento de recepción de material la capacidad normal se calcula multiplicando el número de empleados que trabajan en dicho departamento por su jornada laboral mensual, restándole, a continuación, a dicho producto el tiempo no productivo o de descansos. A continuación, se divide el coste total de dicho departamento entre la capacidad normal y se obtiene el coste por unidad de tiempo (generalmente, coste por minuto). A continuación, los costes son asigna-

Tabla I

Ejemplo de aplicación del coste basado en las actividades en un almacén

\begin{tabular}{|l|c|c|c|c|}
\hline \multicolumn{1}{|c|}{ Actividad } & \% de tiempo & $\begin{array}{c}\text { Coste de } \\
\text { la actividad (€) }\end{array}$ & $\begin{array}{c}\text { Cantidad } \\
\text { del inductor ( } € \text { ) }\end{array}$ & Coste inductor \\
\hline Asignar camiones a punto de descarga & 5 & 7.500 & 200 & $37,5 € /$ entrega \\
\hline Introducir información general de entrega & 10 & 15.000 & 200 & $75 € /$ entrega \\
\hline Descargar pallets de camión & 25 & 37.500 & 2.000 & $18,75 € /$ pallet \\
\hline Puntear albarán de entrega con pallet & 5 & 7.500 & 2.000 & $3,75 € /$ pallet \\
\hline $\begin{array}{l}\text { Introducir datos en el ordenador para obtener al- } \\
\text { barán de entrada y etiquetas }\end{array}$ & 5 & 7.500 & 2.000 & $3,75 € /$ pallet \\
\hline Poner etiquetas de identificación de pallets & 5 & 7.500 & 2.000 & $3,75 € /$ pallet \\
\hline Transporte y ubicación en estanterías & 45 & 67.500 & 2.000 & $33,75 € /$ pallet \\
\hline Total (Suma) & 100 & 150.000 & & \\
\hline
\end{tabular}


Figura 3

Etapas del ABC y del TDABC

A) Etapas del $A B C$

I. Identifica las actividades

2. Asigna los costes indirectos a las distintas actividades a través de los inductores de coste

3. Identifica los inductores de cada actividad

4. Calcula el coste de los inductores dividiendo el coste total de cada actividad entre su volumen de actividad normal

5. Multiplica el coste del inductor por los inductores consumidos para obtener el coste de los objetos de coste (productos, clientes, etc.)

B) Etapas delTDABC

I. Identifica las actividades que son realizadas con los mismos medios para constituir los «grupos de recursos»)

2. Estima los recursos consumidos por cada «grupo de recursos»

3. Estima la capacidad normal de cada grupo de recursos en términos de horas de trabajo

4. Calcula los costes unitarios de los inductores (el más habitual es el minuto de trabajo) de cada grupo de recursos, dividiendo el coste de los recursos consumidos entre la capacidad normal

5. Para cada tarea, determina el tiempo necesario de acuerdo con sus características

6. Para valorar cada tarea, multiplica el coste unitario de los recursos por el tiempo necesario para llevarla a cabo

Fuente: Everaert et al. (2008)

dos a cada pedido de compra multiplicando el coste por unidad de tiempo por el tiempo necesario para completar las operaciones de recepción de los materiales.

Considerando el ejemplo de la Tabla I, el coste total del departamento de recepción de materiales (costes de personal, amortizaciones, suministros y otros) asciende a $150.000 €$ al año, lo que corresponde a una capacidad normal de 7.800 horas (80\% de la capacidad teórica de 37,5 horas a la semana por 52 semanas y 5 empleados). El coste por minuto de este grupo de recursos asciende a 0,32 €. Si el tiempo de una entrada de materiales con paletas homogéneas es de 24 minutos, el coste ascenderá a 7,68 €. En cambio, si la entrada de materiales está formada por paletas no homogéneas el tiempo será de 56 minutos y, por lo tanto, el coste será de 17,92 euros.
La ventaja deITDABC sobre el $A B C$ radica en la estimación del tiempo. El uso del parámetro tiempo como principal inductor de costes permite al sistema de costes TDABC evitar la compleja fase de asignar los costes de los recursos a las actividades, antes de vincularlos a los objetos de coste. El tiempo de realización de una actividad es estimado para cada caso concreto (mediante cronómetro, entrevistas a las personas, etc.). En el modelo convencional de ABC, el responsable de costes pide a las personas que respondan a una serie de cuestionarios sobre cómo reparten su tiempo entre las actividades que realizan. En TDABC las encuestas se abordan de forma diferente. El responsable de costes solicita el tiempo necesario para realizar los pasos concretos de un proceso. Por ejemplo, al analizar el proceso de introducir un pedido de compras, el responsable de TDABC solicita el tiempo que supone dar de alta al proveedor y el tiempo de procesar cada línea del pedido. 
En aquellos casos en los que el tiempo necesario para llevar a cabo una actividad depende a la vez de varios inductores, se modela una ecuación de tiempo (time equation). Una ecuación de tiempo es una ecuación matemática que expresa el tiempo necesario para llevar a cabo una actividad en función de varios inductores como se muestra en la ecuación I.

Tiempo del proceso = suma de tiempos de actividades individuales $=$

$$
\begin{array}{r}
=\left(\beta_{0}+\beta_{1} \cdot X_{1}+\beta_{2} \cdot X_{2}+\beta_{3} \cdot X_{3}+\ldots+\beta_{i} \cdot X_{i}\right)= \\
=\beta_{0}+\Sigma \beta_{i} \cdot X_{i}
\end{array}
$$

Donde $\beta_{0}$ es el tiempo estándar para realizar la actividad básica.

$\beta_{\mathrm{i}}$ es el tiempo calculado para la actividad incremental $i$.

$X_{i}$ es la cantidad de actividad incremental $i$.

Continuando con el ejemplo, el tiempo de introducir en el ordenador un pedido es de I minuto por línea de pedido, al cual es necesario añadir 5 minutos adicionales si el proveedor no está dado de alta previamente en la base de datos; su expresión mediante una ecuación de tiempo se muestra en la ecuación 2.

Tiempo de procesar un pedido de compra $=$

$$
=1 X_{1}+5 X_{2} \text { si el proveedor es nuevo }
$$

Donde $X_{1}$ es el número de líneas del pedido y $X_{2}$ vale uno si el proveedor es nuevo y cero si no lo es.

Por el momento, se han realizado pocos trabajos de investigación sobre la aplicación práctica delTDABC (De la Villarmois y Levant, 2007). Kaplan y Anderson (2004) citan más de 100 aplicaciones reales, pero sólo existe una breve información sobre dos casos ejecutados. Everaert et al. (2008) describen el caso de una empresa distribuidora.

\section{Análisis de un caso}

A continuación se presenta un caso práctico que ilustra las diferencias entre el método TDABC y el $A B C$ original. Permite valorar la mayor o menor complejidad de su aplicación y la bondad de sus resultados. El ejemplo es suficientemente completo para poder apreciar las técnicas del sistema de costes TDABC y a la vez resulta simple para facilitar su comprensión.

Una empresa fabrica y comercializa componentes plásticos para el sector de automoción. Los produc- tos que fabrica son los tiradores traseros de maletero que son los elementos que están situados en la parte posterior del maletero que sirven para la apertura del mismo. El proceso de producción consta de tres fases:

- Primero, se parte de la materia prima base, plástico granulado, y se realiza el proceso de inyección de las piezas.

- A continuación, las piezas se envían a la instalación de pintura donde se les aplica una capa de pintura del color correspondiente.

- En tercer lugar, las piezas pintadas pasan a una célula de montaje.

Para el análisis de costes solo nos centraremos en el proceso de montaje. Se trata de una célula de montaje donde se ensamblan 3 tipos diferentes de tiradores que denominaremos tirador tipo A, tirador tipo B y tirador tipo C.

El proceso de montaje consiste en ensamblar una serie de elementos (interruptor, espuma, clip y tornillos) a las piezas principales que llegan provenientes de la sección de pintura. El interruptor realiza la función de apertura del maletero del automóvil mediante su accionamiento. Está situado en la parte central de la pieza y está sujeto con unos tornillos. El sello de espuma consiste en una larga tira de espuma plástica que se pega a lo largo del contorno del tirador. Su función es el sellado del componente frente a los elementos de la intemperie (agua, humedad y polvo) de manera que éstos no puedan penetrar dentro de la pieza. El clip de plástico y tornillos son los elementos necesarios para el ensamblado del tirador en el coche en la planta de montaje de automóviles, es decir en la planta del cliente. Dependiendo del modelo de tirador el número de tornillos varía.

En la Tabla 2 se indican los tiempos necesarios para el montaje de los tiradores. Estos tiempos han sido medidos observando a los trabajadores de la célula de montaje (son el resultado de una media ponderada aproximada de todas las muestras tomadas a lo largo de diferentes días y distintos operarios).

\section{I. Cálculo de costes mediante $A B C$}

Las etapas abordadas en el sistema de costes ABC han sido la definición de actividades y costes y el cálculo de costes de los productos. 
Tabla 2

Tiempos de montaje de los tiradores

\begin{tabular}{|c|c|}
\hline Tipo de tirador & Tiempo (segundos) \\
\hline Modelo A & 140 \\
\hline Modelo B & 100 \\
\hline Modelo C & 80 \\
\hline
\end{tabular}

\subsubsection{Definición de actividades y costes}

Para la aplicación del sistema de costes $A B C$ se han entrevistado a las personas implicadas (que pertenecen a distintos departamentos) para que describan el tipo de actividades que realizan, cuanto tiempo dedican, los recursos que se consumen y las medidas de actividad o inductores. Toda esta información se muestra en la Tabla 3. Los datos están referidos a un mes.

\subsubsection{Cálculo de costes de los productos mediante $A B C$}

Con la información de las tablas 2 y 3 se pueden obtener los costes de cada uno de los tipos de tiradores (verTabla 4).

\subsection{Cálculo de costes mediante TDABC}

Las etapas abordadas en el sistema de costes TDABC son:

I. ${ }^{\text {a }}$ Desarrollo de las ecuaciones de tiempo

2. ${ }^{\text {a }}$ Coste por unidad de tiempo de los grupos de recursos (reposición de materiales y control de calidad)

$3^{\mathrm{a}}$ Estimación de costes de las actividades

Tabla 3

Actividades, costes e inductores para una línea de montaje

\begin{tabular}{|c|c|c|c|c|c|}
\hline Actividad & $\%$ de tiempo & $\begin{array}{c}\text { Coste de } \\
\text { la actividad } \\
\text { (€) }\end{array}$ & Inductor & $\begin{array}{l}\text { Número de } \\
\text { inductores }\end{array}$ & $\begin{array}{l}\text { Coste } \\
\text { inductor }\end{array}$ \\
\hline $\begin{array}{l}\text { Reposición componentes a la línea de } \\
\text { montaje }\end{array}$ & $15 *$ & 3.000 & $\begin{array}{l}\text { Unidades } \\
\text { montadas }\end{array}$ & 5.000 & $\begin{array}{l}0,6 \\
€ / \text { unidad }\end{array}$ \\
\hline Montaje & $100 * *$ & 24.960 & $\begin{array}{l}\text { Horas de mano } \\
\text { de obra }\end{array}$ & 156 & $\begin{array}{l}160 \\
€ / \text { hora }\end{array}$ \\
\hline Control de calidad* & 12,5 **** & 4.000 & $\begin{array}{l}\text { Unidades } \\
\text { montadas }\end{array}$ & 5.000 & $\begin{array}{l}0,8 \\
€ / \text { unidad }\end{array}$ \\
\hline
\end{tabular}

* Tanto por ciento de la jornada que un reponedor dedica a esta línea (el resto del tiempo lo dedica a otras secciones de la empresa)

** Un operario de la célula dedica toda la jornada a montaje

**** Tanto por ciento de la jornada que un empleado de control de calidad dedica a esta línea

****** 2.000 unidades del tirador A, 2.000 unidades del tirador B y 1.000 unidades del tirador C

Tabla 4

Coste de los productos mediante $A B C$

\begin{tabular}{|c|c|c|c|c|}
\hline & \multicolumn{3}{|c|}{ ACTIVIDADES } & \multirow{2}{*}{$\begin{array}{c}\text { Actividades } \\
\text { Total } \\
\text { (€lunidad) }\end{array}$} \\
\hline & $\begin{array}{l}\text { Reposición } \\
\text { (€lunidad) }\end{array}$ & $\begin{array}{l}\text { Montaje } \\
\text { (€lunidad) }\end{array}$ & $\begin{array}{c}\text { Control de calidad } \\
\text { ( } € / \text { lunidad })\end{array}$ & \\
\hline Tirador A & 0,6 & $(140 / 3600) \times 160=6,22$ & 0,8 & 7,62 \\
\hline Tirador B & 0,6 & $(100 / 3600) \times 160=4,44$ & 0,8 & 5,84 \\
\hline Tirador C & 0,6 & $(80 / 3600) \times 160=3,55$ & 0,8 & 4,95 \\
\hline
\end{tabular}




\subsubsection{Desarrollo de las ecuaciones de tiempo}

En este apartado se desarrollan las ecuaciones de tiempo para las actividades de reposición de materiales y control de calidad.

\section{Actividad de reposición de materiales}

Esta actividad comprende todas las tareas relativas al proceso de reposición de materiales. Dependiendo del tipo de producto, el tiempo que supone reponer los materiales es diferente. Para el tirador A, el tiempo es de 9 minutos. Para el tirador B, el tiempo es de 6 minutos y por último, para el tirador $C$, el tiempo es de 4 minutos. Se puede plantear como la ecuación 3.

Tiempo de reposición (en minutos) $=$

$$
=9 x_{1}+6 x_{2}+4 x_{3}
$$

Donde

$X_{1}=1$, si son los componentes del tirador A. En caso contrario, $X_{1}=0$.

$X_{2}=1$, si es tirador $B$. En caso contrario, $X_{2}=0$.

$X_{3}=1$, si es tirador $C$. En caso contrario, $X_{3}=0$.

El número de veces (en un mes) que se repone material para cada uno de los tiradores se muestra en la Tabla 5.
Tabla 5

Número de veces al mes que se repone material por tipo de producto

\begin{tabular}{|c|c|}
\hline Tirador A & 80 \\
\hline Tirador B & 50 \\
\hline Tirador C & 30 \\
\hline
\end{tabular}

Actividad de control de calidad

El tiempo que lleva efectuar el control de calidad de cada tirador depende del número de puntos a revisar o controlar. En el caso del tirador $\mathrm{A}$ el número de puntos son 2, en el caso del tirador B son 4 y el tirador C, 6. Esto se podría implementar según la ecuación 4.

Tiempo control calidad (en minutos) $=0,05 X$

Donde

$X=$ número de puntos a revisar (2 en tirador $A, 4$ en tipo B y 6 en $C$ )

\subsection{Coste por unidad de tiempo de los grupos de recursos}

El coste por unidad de tiempo (minuto) para cada grupo de recursos se calcula según muestran las tablas 6 y 7. La capacidad disponible por mes (en horas) está basada en 40 horas trabajadas por semana

Tabla 6

Coste por unidad de tiempo de la actividad de reposición de materiales

\begin{tabular}{|c|l|c|c|}
\hline I. & Capacidad disponible por semana & $40 \mathrm{~h} \times 0,15$ personas & $6,0 \mathrm{~h}$ \\
\hline 2. & Capacidad normal por mes & $80 \% \times 6 \mathrm{~h} \times 4=$ & $19,2 \mathrm{~h}$ \\
\hline 3. & Coste total (por mes) & & $3.000 €$ \\
\hline 4. & Coste por unidad de tiempo & & $156,25 € / \mathrm{h}$ \\
\hline \multirow{2}{*}{} & Coste por hora & $3.000 € / 19,2 \mathrm{~h}=$ & $2,60 € / \mathrm{min}$ \\
\cline { 2 - 4 } & Coste por minuto & $156,25 € / \mathrm{h} / 60 \mathrm{~min} / \mathrm{h}=$ & \\
\hline
\end{tabular}

Tabla 7

Coste por unidad de tiempo de la actividad de control de calidad

\begin{tabular}{|c|l|c|c|}
\hline I. & Capacidad disponible por semana & $40 \mathrm{~h} \times 0,12$ personas & $4,8 \mathrm{~h}$ \\
\hline 2. & Capacidad normal por mes & $80 \% \times 4,8 \mathrm{~h} \times 4=$ & $15,36 \mathrm{~h}$ \\
\hline 3. & Coste total (por mes) & & $4.000 €$ \\
\hline 4. & Coste por unidad de tiempo & & $260,42 € / \mathrm{h}$ \\
\hline \multirow{2}{*}{} & Coste por hora & $4.000 € / 15,36 \mathrm{~h}=$ & $4,34 € / \mathrm{min}$ \\
\cline { 2 - 4 } & Coste por minuto & $260,42 € / \mathrm{h} / 60 \mathrm{~min} / \mathrm{h}=$ & \\
\hline
\end{tabular}


y persona. Se ha estimado la capacidad normal como el $80 \%$ de la capacidad teórica.

\subsubsection{Cálculo de costes de los productos}

Según la tabla 5 , en un mes se reponen 80 veces los componentes del tirador A, 50 veces los del tirador B y 30 veces los componentes del tirador C. Con los coeficientes de la ecuación 3, podemos determinar el tiempo de reposición para cada tipo de tirador y el coste de reposición por unidad de artículo, multiplicando el tiempo dedicado a cada uno de los tiradores por el coste por unidad de tiempo y dividiendo entre la producción realizada (Tabla 8).

De forma análoga, aplicando la ecuación 4, se obtiene el coste de control de calidad para cada uno de los tiradores (Tabla 9).
Añadiendo al coste de reposición e inspección el coste de montaje (que es el mismo que con el sistema de costes $A B C$ ) se obtiene el coste de cada uno de los productos mediante TDABC (Tabla 10).

\section{Conclusiones e implicaciones para la gestión}

El caso de estudio muestra una serie de ventajas e inconvenientes en la aplicación del método TDABC. Los resultados de este trabajo muestran como el sistema de costes TDABC proporciona una información de costes más precisa que el sistema de costes $A B C$ : El modelo $A B C$ proporciona un coste único en la actividad de reposición de materiales y en el control de calidad. El modelo TDABC distingue si los materiales son para el tirador I, para el tirador 2 o para el tirador 3. Lo mismo sucede con la actividad de

Tabla 8

Cálculo del coste de reposición por artículo

\begin{tabular}{|c|c|c|}
\hline Tipo de producto & $\begin{array}{c}\text { Tiempo (minutos) de reposición } \\
\text { de materiales }\end{array}$ & $\begin{array}{c}\text { Coste de reposición por artículo } \\
\text { (धltirador) }\end{array}$ \\
\hline Tirador A & $9 \times 80=720$ & $720 \times 2,60 / 2000=0,936$ \\
\hline Tirador B & $6 \times 50=300$ & $300 \times 2,60 / 2000=0,390$ \\
\hline Tirador C & $4 \times 30=120$ & $120 \times 2,60 / 1000=0,312$ \\
\hline
\end{tabular}

Tabla 9

Cálculo del coste de control de calidad por artículo

\begin{tabular}{|c|c|c|}
\hline Tipo de producto & Tiempo (minutos) de inspección & $\begin{array}{c}\text { Coste de control por artículo } \\
(€ / \text { tirador) }\end{array}$ \\
\hline Tirador A & $0,10 \times 2000=200$ & $200 \times 4,34 / 2000=0,434$ \\
\hline Tirador B & $0,20 \times 2000=400$ & $400 \times 4,34 / 2000=0,868$ \\
\hline Tirador C & $0,30 \times 1000=300$ & $300 \times 4,34 / 1000=1,302$ \\
\hline
\end{tabular}

Tabla 10

Coste de los productos mediante TDABC

\begin{tabular}{|c|c|c|c|c|}
\hline Producto & $\begin{array}{c}\text { Reposición } \\
(€ / \text { tirador })\end{array}$ & Montaje( $€ /$ tirador $)$ & Calidad (€/tirador) & Total ( $€ /$ tirador) \\
\hline Tirador A & 0,936 & $(\mid 40 / 3600) \times 160=6,22$ & 0,434 & 7,590 \\
\hline Tirador B & 0,390 & $(\mid 00 / 3600) \times 160=4,44$ & 0,868 & 5,698 \\
\hline Tirador C & 0,312 & $(80 / 3600) \times 160=3,55$ & 1,302 & 5,164 \\
\hline
\end{tabular}


control de calidad, en la que el coste es diferente ya que el mismo depende del número de los puntos a revisar.

Aparentemente, ha sido más complejo recopilar la información necesaria para aplicar el método TDABC, pero para poder llegar al mismo detalle en $A B C$ sería necesario descomponer cada actividad en numerosas subactividades y estimar los recursos consumidos por cada una de ellas, lo que sería aún más complejo. Además, este último proceso de reparto de costes entre las subactividades en $A B C$ tiene un importante grado de subjetividad.

TDABC, al igual que $A B C$, es un sistema de costes completos, pues al final se acaban repartiendo los costes indirectos de las secciones o grupos en base a algún criterio. En el caso de TDABC su inductor es básicamente el tiempo de trabajo.

Otra conclusión alcanzada es que, por extensión, con el sistema TDABC se pueden modelar las actividades de la empresa con un número no muy alto de ecuaciones de tiempo. Si se quisiera desarrollar los supuestos de variabilidad en ABC sería necesario desglosar cada actividad en varias subactividades y su número crecería sustancialmente. Por otro lado, el modelado de las ecuaciones de tiempo es una fuente de error del sistema TDABC: si la ecuación no se ajusta a la realidad, los costes también serán falsos.

El sistema TDABC trabaja con tiempos estandarizados por lo que se requiere disponer de dichas mediciones y mantenerlas al día. Las técnicas de métodos y tiempos son habituales en las empresas industriales, pero se intuye que en el sector servicios, con trabajos normalmente menos estandarizados, su aplicación puede ser difícil.

La metodología TDABC, al trabajar con inductores de tiempo, proporciona un análisis de la capacidad. TDABC informa continuamente de la capacidad infrautilizada y sobreutilizada de sus empleados. Esto ayuda a los gestores a tomar decisiones sobre posibles cambios en recursos (personas) de un área a otra para alcanzar un mejor equilibrio de las capacidades demandadas y suministradas por los departamentos. Como inconveniente, el cálculo parte de un nivel de actividad que se denomina normal. En función de donde se fije éste, se obtendrán unos u otros resultados.

El sistema $A B C$ informa de los costes de las actividades pero no permite saber como se utiliza el tiempo en su ejecución, siendo un parámetro muy importante para la mejora continua de dichas actividades y la medida de la productividad como un indicador de la eficiencia productiva global, objetivo último de sistemas como lean manufacturing o la teoría de las limitaciones.

El sistema TDABC más que en actividades se centra en procesos, con lo que el modelo resulta más manejable. Presupone que los trabajos en la empresa son repetitivos (de otro modo el modelado no sería de gran utilidad) y con ellos los procesos no cambian, por lo que pueden ser modelados con las ecuaciones de tiempo.

Aunque son aún pocos los casos de empresas que aplican el sistema TDABC y lógicamente menos los recogidos en la literatura científica, el sistemaTDABC se muestra especialmente útil en las empresas donde existe diversidad de productos y complejidad de procesos productivos. Esto es especialmente útil en tareas donde el uso de la mano de obra es intensivo y en el sector servicios, donde además de ser fundamental el trabajo humano, las tareas son muy variadas. Así por ejemplo, Carrasco y Ponce (2008) analizan la mejora de la eficiencia en una central logística. Los costes de operación en dicha planta podrían calcularse mediante el sistema TDABC como muestran distintos trabajos de aplicación al sector logístico (Everaert et al, 2008). De modo similar, en un sistema de recogida y tratamiento de residuos (Ortega Mier et al., 2008), los costes de las plantas de recuperación podrían adaptarse al sistema TDABC.

En empresas industriales donde los productos no son nunca iguales y la mano de obra es importante, por ejemplo, las empresas de roca ornamental (Vintró y Fortuny, 2010) el TDABC puede calcular los costes con mayor facilidad que otros sistemas.

Esto es especialmente útil cuando el conocimiento correcto de los costes por la existencia de poco margen se combina con la variabilidad de productos y procesos y donde el ensamblaje manual es importante, como puede ser el caso de los fabricantes de componentes para el automóvil (Llorente, 20 l0). En dicho caso elTDABC puede ser una herramienta virtualmente imprescindible.

\section{Bibliografia}

BAGUER, A., y ZARRAGA, M. (2002). Dirige: manual de conceptos prácticos y necesarios para la gestión empresarial, Madrid: Ediciones Díaz de Santos. 
CARRASCO, R. y PONCE, E. «Mejora de la eficiencia de una central logística mediante el resideño del reaprovisionamiento de la zona de picking». Dirección y Organización, no 36, 2008, pp. 73-81.

DE LAVILLARMOIS, O., y LEVANT,Y. (2007). «Le time-driven $A B C$ : la simplification de I'évaluation des coûts par le recours aux équivalents -un essai de positionnement». Revue Finance Contrôle Stratégie, I0 (I), pp. |49-182.

EVERAERT, P., BRUGGEMAN, W., SARENS, G., ANDERSON, S. R., y LEVANT,,Y. (2008). «Cost modelling in logistics using time-driven $A B C$. Experiences from a wholesaler». International Journal of Physical Distribution \& Logistics Management, 38 (3), pp. I72-।91.

GOLDRATT E. M., y COX, J. (2005). La meta. Un proceso de mejora continua, 3a edición revisada en castellano, Madrid: Ediciones Diaz de Santos.

IGLESIAS SANCHEZ, J.L. (2004). «Importancia de la Teoría de las Limitaciones en la Contabilidad de Gestión». Revista iberoamericana de contabilidad de gestión, 4,, pp. 207-225.

KAPLAN, R. S. (1983). «Measuring manufacturing performance: a new challenge for managerial accounting research». The accounting review, 58 (4), pp. 686-705.

KAPLAN, R. S. (1984). «Yesterday's accounting undermines production». Harvard Business Review, 62 (July-August), pp. 95-|01.

KAPLAN, R.S., y ANDERSON, S. (2004). «Time-Driven Activity-Based Costing». Harvard Business Review, 82 ( I I), pp. |3|-| 38.

KAPLAN, R.S., y ANDERSON, S. (2007). Time-Driven Activity-Based Costing. A simpler and more powerful path to higher profits, Boston: Harvard Business School Press.
KAPLAN, R.S., y COOPER, R. (1 988). «Measure Costs Right; Make The Right Decisions». Harvard Business Review, 66 (5), pp. 96- 103.

LLORENTE, F. «Innovación tecnológica en la industria automovilística catalana». Dirección y Organización, $n^{\circ} 40$, 2010 , pp. 14-27.

MALLO, C., y JIMENEZ, M. A. (2000). Contabilidad de costes, Madrid: Ediciones Pirámide, pp. 405-428.

MARIN, F., y DELGADO, J. (2000). «Las técnicas justo a tiempo y su repercusión en los sistemas de producción». Economía industrial, 331, pp. 35-4l.

ORTEGA-MIER, M., DELGADO, H.y GARCIA, A. «Uso de algoritmos genéticos para resolver el modelo determinista y estocástico para el diseño de una red de recogida de residuos»». Dirección y Organización, nº 35, 2008, pp. 15-22.

RIGBY, D.K. (2003). Management Tools 2003, Boston: Bain \& Company Publishing.

RIPOLL FELIU, V. M., y TAMARIT AZNAR, M. C. (1996). «Sistematización de la filosofía JIT en el ABC/ABM». Técnica contable, 48 (569), pp. 313-326.

TATIKONDA, M.V. (1988). «Just-in-time and modern manufacturing environments: implications for cost accounting». Production and inventory management journal, First quarter, pp. I-5.

TIRADO, P. (2003). Gestión de costes y mejora continua, Bilbao: Desclée de Brouwer.

VINTRÓ, C.Y FORTUNY, J. «Estudio empírico de las prácticas de Responsabilidad Social Corporativa en la minería de áridos en Cataluña». Dirección y Organización, $n^{\circ} 42,2010$, pp. 16-23. 